\title{
Design and Performance Analysis of a CD Nozzle for Evacuating Emissions from the Subway Tunnels
}

\author{
Nallode C*, Rakesh C, Ahmed J and Ganesha Prasad MS
}

Department of Mechanical Engineering, New Horizon College of Engineering, Bengaluru, India

\begin{abstract}
Tunnels have very severe air quality constraints that typically require good ventilation system. The process of removing air from any space by mechanical means is known as evacuation. Evacuation of exhaust and smoke in case of fire emergencies is very necessary to bring in fresh air as it leads to pressure and temperature build up which causes discomfort to the passengers travelling especially in long tunnels. Generally, in longitudinal ventilation, a jet blower fan enclosed in a cylindrical casing is used for ventilation. These jet fans accelerate the air by imparting it an impulse in the desired direction. The main aim is to optimize the design and replace the jet blower fan cylindrical enclosure by a CD Nozzle. The suction fan along with rotor is exactly placed at the throat of the nozzle. The modeling is done using CATIA V5 software and CFD flow and modal analysis is carried out using ANSYS V 15.0. Various parameters like pressure, temperature, density, humidity and mass flow rate of air are measured and compared with the conventional model by experimentation and the results are validated.
\end{abstract}

Keywords: CD Nozzle; Evacuation; Longitudinal ventilation; Jet fan; Computational Fluid Dynamics (CFD)

\section{Introduction}

\section{Tunnel ventilation}

Tunnels may require ventilation for a variety of reasons - For example to ensure an adequate air quality, to control the spread of smoke in case of fire, or to reduce temperatures to acceptable limits. The function of the ventilation relates to the type of tunnel in question. Vehicular tunnels (road, rail and metro) where, due to the exhaust gases, the air is severely contaminated with carbon monoxide, nitrogen oxide and LPG (liquefied petroleum gas). Depending on the concentration and the length of time spent in the tunnel there is a great danger for the passengers travelling as it may cause suffocation. CO is easily absorbed by Haemoglobin in human blood and can inhibit oxygen delivery to body and can cause adverse health effects varying from headache to death. Therefore, the polluted air needs to be extracted quickly and safely. However, the duct systems which are often used for this take-up a lot of space and often cross other services such as electrical or water lines. So, a ductless system is used which is reflected in the investment and installation costs. A number of alternative tunnel ventilation systems are available, including transverse, semi-transverse and longitudinal. Depending on fire risk mitigation and the maintenance of adequate air quality, longitudinal ventilation systems can be installed in much longer tunnels [1-5].

Jet blower fans - a type of longitudinal ventilation also known as impulse or induction fans - support the natural flow between the supply air and extract air zones [6-9]. They provide motion in regions with low air speeds, thus guaranteeing the daily ventilation requirement for all areas. Since jet fans are only installed at particular points, they take up less than $0.5 \%$ of the ceiling area. This leaves room for other technical installations and the visibility across the tunnel decks is improved. Jet fans are usually axial fans enclosed in a cylindrical casing.

\section{CD nozzle}

De-Laval nozzle (Convergent - Divergent nozzle) is a tube that is pinched in the middle, making a carefully balanced, asymmetric hour glass shape. It is used to accelerate a hot, pressurized gas passing through it to a supersonic speed, and upon expansion, to shape the exhaust flow so that the heat energy propelling the flow is maximally converted into directed kinetic energy. Because of this, the nozzle is widely used in some types of steam turbines, it is an essential part of the modern rocket engine, and it also sees use in supersonic jet engines (Figures 1-6).

\section{Objective of the Research}

The main aim of this research is to optimize the design of the enclosure of the jet fan and increase the performance of it.

The objectives of this research are:

1. Design, fabricate and analyze the performance of a C-D nozzle with a rotor and fan.

2. Rotor and fan to be mounted inside the $\mathrm{CD}$ nozzle at the throat area.

3. Replace the cylindrical enclosure by CD Nozzle.

4. Analyze the performance of both by comparison.

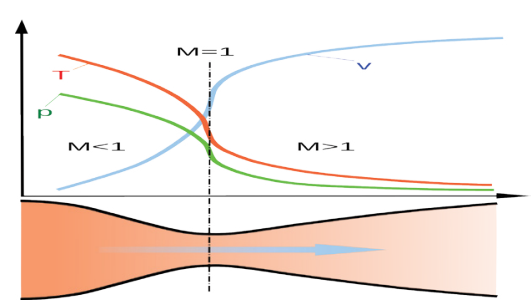

Figure 1: CD Nozzle.

*Corresponding author: Nallode C, Department of Mechanical Engineering, New Horizon College of Engineering, Bengaluru-560103, India, Tel: 098805 34935; E-mail: charan.wkf@gmail.com

Received October 30, 2017; Accepted November 10, 2017; Published November 15,2017

Citation: Nallode C, Rakesh C, Ahmed J, Ganesha Prasad MS (2017) Design and Performance Analysis of a CD Nozzle for Evacuating Emissions from the Subway Tunnels. J Appl Mech Eng 6: 293. doi: 10.4172/2168-9873.1000293

Copyright: @ 2017 Nallode $\mathrm{C}$, et al. This is an open-access article distributed under the terms of the Creative Commons Attribution License, which permits unrestricted use, distribution, and reproduction in any medium, provided the original author and source are credited. 


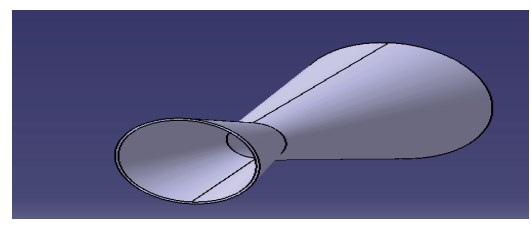

Figure 2: CD Nozzle model.

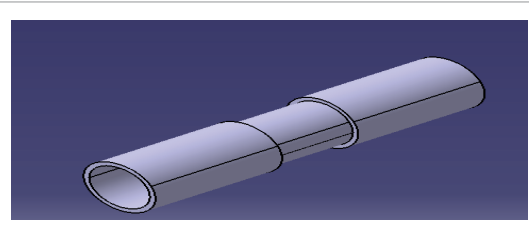

Figure 3: Cylindrical enclosure.

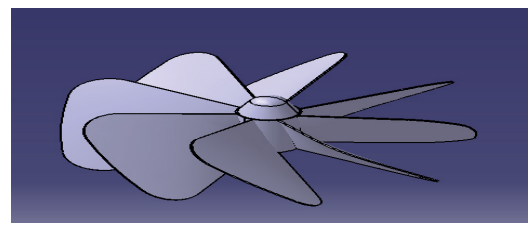

Figure 4: Axial fan.

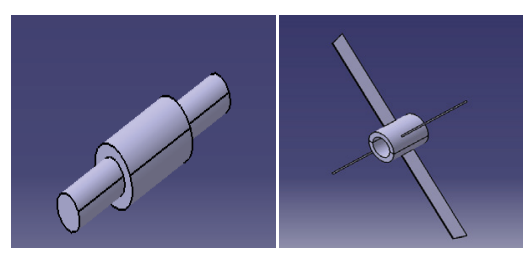

Figure 5: Rotor and fan mount.

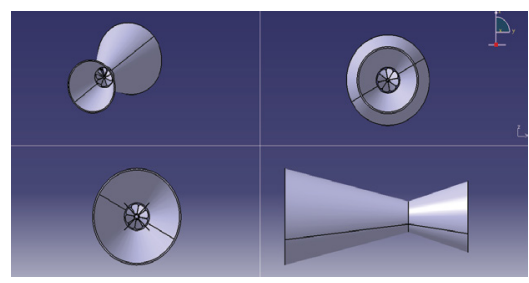

Figure 6: Assembled CD nozzle model.

5. Validate the results through experimentation.

\section{Methodology}

- Firstly, the case study of the jet fan available and the CD Nozzle is done.

-The design parameters and the boundary conditions are deter mined.

- The designing of the $\mathrm{CD}$ nozzle is done for desired outlet conditions

- The modeling for both CD nozzle and cylindrical enclosure is done using the CATIA V5 software.

- Then the meshing is done using ANSYS V.15 software.

- The fluid flow analysis is done using Ansys FLUENT to obtain various velocity, pressure, Mach number and temperature contours.
- The results are then validated by comparing both the models and as well as by experimentation.

\section{Design parameters}

There are various parameters that considered are explained below. The inlet conditions include the inlet pressure $\left(\mathrm{p}_{\mathrm{i}}\right)$, inlet temperature $\left(T_{i}\right)$, gamma $(\gamma)$ and inlet diameter $\left(D_{i}\right)$. These parameters are fixed from various case studies and literature survey. The parameter ranges for the inlet studied from various studies are given below (Figures 1-6):

\section{Nozzle}

Inlet pressure is in the range of 2 bars.

Inlet diameter is in the range of $282 \mathrm{~mm}$.

Throat diameter $=122 \mathrm{~mm}$.

Outlet diameter $=382 \mathrm{~mm}$.

Inlet temperature specified for the nozzle $=303 \mathrm{~K}$.

gamma $(\gamma)=1.4$.

Velocity of air (assumed) $=10 \mathrm{~m} / \mathrm{sec}$.

Density of air $=1.225 \mathrm{~kg} / \mathrm{m}^{3}$.

\section{Cylindrical Duct}

Inlet pressure is in the range of 2 bars.

Cylindrical diameter is in the range of $122 \mathrm{~mm}$.

Inlet temperature specified for the nozzle $=303 \mathrm{~K}$.

Gamma $(\gamma)=1.4$.

Mass flow rate (assumed) $=1.22 \mathrm{~m}^{3} / \mathrm{sec}$.

\section{Fan}

Fan blade diameter $=100 \mathrm{~mm}$.

Fan hub diameter $=15 \mathrm{~mm}$.

Fan blade thickness $=20 \mathrm{~mm}$.

\section{Rotor}

Rotor length $=100 \mathrm{~mm}$.

Rotor diameter $(\mathrm{mid})=25 \mathrm{~mm}$.

Rotor diameter $($ edge $)=15 \mathrm{~mm}$.

\section{Fixutre (Fan)}

Fixture diameter (rotor mount) $=15 \mathrm{~mm}$

Fixture thickness $=30 \mathrm{~mm}$.

Fixture arms (four) $=60 \mathrm{~mm}$.

\section{Modeling}

The modeling is done by using CATIA V5. The diagrams below show various geometries designed.

\section{Assembly}

The models created are then assembled and the figure below shows the multi-view of the CD nozzle and cylindrical model (Figures 6 and 7).

\section{Meshing}

The mesh generation process depends on the model prepared in the 
modeling software. Fine meshing is done for better and accurate results as shown below in Figures 8 and 9.

\section{Input and boundary conditions}

This is shown in Table 1.

\section{Results and Discussion}

The contours of total pressure and velocity-Mach number for a CD Nozzle case are shown below in the Figures 10 and 11.

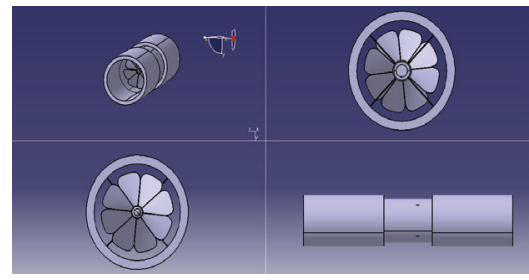

Figure 7: Assembled cylindrical model.

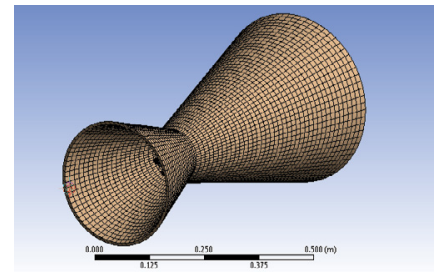

Figure 8: Meshing of CD model.

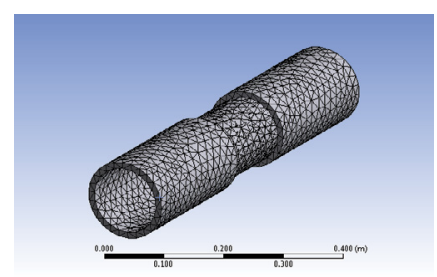

Figure 9: Meshing of cylindrical model.

\begin{tabular}{|c|c|c|c|c|}
\hline Cases & $\begin{array}{c}\text { Inlet pressure } \\
\text { (bar) }\end{array}$ & $\begin{array}{c}\text { Inlet Velocity } \\
\text { (m/s) }\end{array}$ & Medium & Inlet Temp (K) \\
\hline Cylindrical & 1 & 10 & Air (Ideal gas) & 303 \\
\hline CD Nozzle & 1 & 10 & Air (Ideal gas) & 303 \\
\hline
\end{tabular}

Table 1: Boundary conditions.

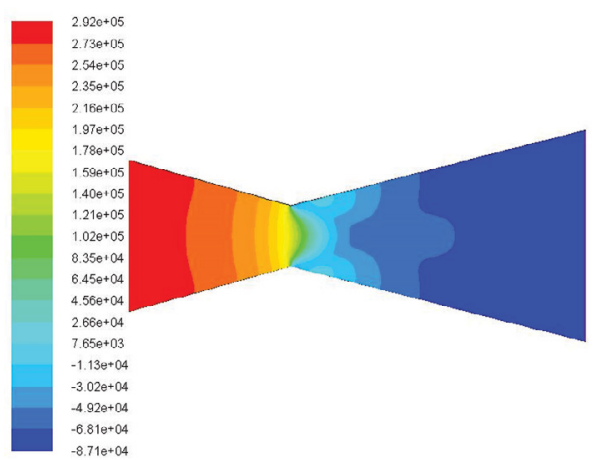

Figure 10: Pressure contour.

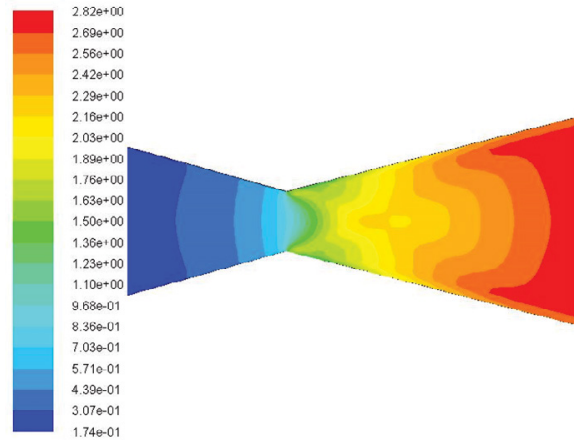

Figure 11: Velocity - mach number cont.

The present study is focused on optimizing the design and performance of jet fan with CD nozzle enclosure. The conventional cylindrical jet fan and the CD Nozzle along with fan, rotor and fixture are modeled and assembled using Catia V5 software. The model is imported to ANSYS CFX software where it meshed and the fluid flow analysis of both the model is done.

\section{Conclusion}

The results obtained analytically are in accordance with theoretical relations used thus validating the results. It can be concluded that the optimization of the design resulted in better performance that is air is accelerated at must faster rate and reduced pressure in the CD Nozzle model than the conventional cylindrical model.

\section{Future Scope}

The work is to be further continued by doing fluid flow analysis for both the models and imparting rotational speed to the fan to accelerate the air at much better rate. The present study focuses only on circular geometry of the CD Nozzle and this work can be further continued by changing the geometry to rectangular and ellipse at the inlet, outlet and at the throat.

\section{Acknowledgments}

We thank Dr. Manjunatha, principal of NHCE and Dr. M.S. Ganesha Prasad, HOD, Department of Mechanical Engineering, NHCE, Bengaluru for their constant support and guidance.

\section{References}

1. Pansari K, Jilani SAK (2013) Analysis of the performance and flow characteristics of convergent divergent (c-d) nozzle. Swami Vivekananda University, Raipur (CG) IJAET 6: 1313-1318.

2. Pandey KM, Member IACSIT, Singh AP (2010) CFD analysis of conical nozzle for mach 3 at various angles of divergence with fluent software. Int J Chemical Eng and Appl.

3. Akande JM, Moshood O (2013) Modelling of okaba underground coal mine ventilation system. The Federal University Of Technology Akure, Ondo State, Nigeria.

4. Swaroopini SA, Kumar GM, Kumar NT (2015) Numerical simulation and optimization of high performance supersonic nozzle at different conical angles. IJRET 4: 2321-7308.

5. Hossain S, Raiyan FM, Hassan JN (2014) Comparative study of supersonic nozzles. IJRET.

6. Gaikwad PA, Padole V (2016) Design and vibration analysis of existing exhaust fan using finite element analysis technique. IJSRD.

7. Herdeen JA, Sullivian PT (2003) The application of CFD for evaluation of dust suppression and auxiliary ventilation systems used with continuous. Miners pp: 293-297. 
Citation: Nallode C, Rakesh C, Ahmed J, Ganesha Prasad MS (2017) Design and Performance Analysis of a CD Nozzle for Evacuating Emissions from the Subway Tunnels. J Appl Mech Eng 6: 293. doi: 10.4172/2168-9873.1000293

Page 4 of 4

8. Pulse Jet Manual (2009) The Technical Handbook and Manuals of Jet fan and jet blower system. Pulse Jet, Malayisa,
9. Ismail M, Rahman MA (2012) Rooftop turbine ventilator: A review and update. 